\title{
Diagnosis and therapy of rare central nervous system infections
}

\author{
Xiao-Kun Qi \\ Department of Neurology, Navy General Hospital of PLA, Beijing 100048, China.
}

\section{INTRODUCTION}

Central nervous system (CNS) infection is one of the most disabling and deadly diseases worldwide. According to the World Health Organization, there were about 700,000 cases of meningitis in 2004, with approximately 340,000 related deaths. ${ }^{[1]}$ CNS infection includes infection with bacteria, viruses, fungi and parasites. In most cases, it is difficult for radiologists and clinicians to make a definitive diagnosis. Therefore, we reviewed all the relevant domestic and international clinical research development.

\section{DIAGNOSIS AND DIFFERENTIAL DIAGNOSIS OF CENTRAL NERVOUS SYSTEM MYCOBACTERIUM TUBERCULOSIS INFECTION}

Central nervous system tuberculosis, accounting for 5\%-10\% of all types of Mycobacterium tuberculosis (MTB) infection, is an extra-pulmonary tuberculosis leading to various complications and high rates of morbidity and mortality. ${ }^{[2]}$ CNS tuberculotic patients may show either general manifestations caused by toxins from $\mathrm{N} /$ med tuberculosis bacilli, such as low-grade fever, night sweats and headache, or the symptoms of encephalitis and meningitis. Major complications of tuberculous meningitis include hydrocephalus, tuberculous vasculitis and cranial nerve palsies. Intracranial tuberculosis includes tuberculoma tuberculosis, tuberculous abscess, tuberculosis encephalitis, and encephalopathy. ${ }^{[3]}$

\begin{tabular}{|l|l|}
\hline \multicolumn{2}{|c|}{ Access this article online } \\
\hline Quick Response Code: & \\
\hline & Website: \\
\hline & Www.nnjournal.net \\
\cline { 2 - 3 } & DOI: \\
\hline
\end{tabular}

The clinical symptoms of tuberculous meningitis are relatively obvious. Among them, headache and fever are the most common symptoms. Besides, the symptoms of tuberculous meningitis, tuberculous meningoencephalitis can also show signs of the brain parenchymal involvement, insanity or cognitive impairment, and signs of specific area damage, such as cranial nerve palsies and epilepsy. Typical characteristic cerebrospinal fluid (CSF) findings of CNS tuberculosis include the following: total white cell counts increase (usually [50-200] $\times 10^{6} / \mathrm{L}, 1000 \times 10^{6} / \mathrm{L}$ in very few cases), and neutrophil predominance presents very early, with lymphocytic-predominant pleocytosis developing later; an elevated CSF protein, typically 1-2 g/L; and a significant reduction in sugar and chloride levels. Tuberculous meningoencephalitis often occurs with atypical clinical features, sometimes with the acute or chronic onset, manifested by fever, neurological symptoms, and nondiagnostic CSF findings. Above all, at present, we need to make a comprehensive judgment-based on both clinical features and image data of the patients. Meningeal enhancement is usually visible at the skull base, lateral fissure, optic chiasma, and brain stem. When CSF protein is increased significantly, computed tomography (CT) images may additionally show enhancement of the suprasellar cistern, optic chiasma cistern, and prepontile cistern due to exudative reaction. Magnetic resonance imaging (MRI) offers a significant advantage for diagnosis of CNS tuberculosis, especially when incorporating magnetization transfer imaging. ${ }^{[3]}$

Nowadays, the typical fever symptom in tuberculoma patients is rare, and CSF changes may not be typical either. The only abnormal finding is the presence of granulomas in brain CT and MRI images, with nodular and rim enhancement and without obvious meningeal enhancement. CNS tuberculoma must sometimes be

Corresponding Author: Prof. Xiao-Kun Qi, Department of Neurology, Navy General Hospital of PLA, No. 6 Fucheng Road, Beijing 100048, China. E-mail: biqxk@sina.com 
distinguished from CNS sarcoidosis, which leads to a significant increase in angiotensin-converting enzyme in the serum and most of the patient's CSF.

Tuberculous meningitis can easily be confused with cryptococcal meningitis, which has similar manifestations, such as fever, headache, and signs of meningeal irritation, but cryptococcal meningitis has higher elevated intracranial pressure, lower CSF chloride, and rarely any red blood cells in the CSF. Cryptococcus meningitis can be confirmed by CSF India ink capsule staining. By contrast, tuberculous meningitis often has red blood cells in the CSF, a greater number of changes in the WBC count, and higher CSF protein.

Although T-SPOT.TB and Xpert MTB/RIF assays have improved the positive detection rate of MTB in CSF, diagnosis of tuberculous meningitis remains difficult. A modified acid fast stain designed by doctors from the Department of Neurology, Xijing Hospital of the Fourth Military Medical University, has significantly improved the positive detection rate of MTB in CSF. ${ }^{[4]}$ However, there is still an urgent need for more effective testing techniques to improve the detection rate of MTB.

One way to diagnose tuberculosis is by antituberculosis treatment itself. For patients in whom a definitive diagnosis is difficult, antituberculosis treatment can be used to diagnose - through observation - any improvement in clinical symptoms and changes in the CSF. For all tuberculotic patients, both standard treatment and a strict antituberculosis treatment regimen are needed; treatment duration is usually $18-24$ months.

\section{DIAGNOSIS AND DIFFERENTIAL DIAGNOSIS OF CENTRAL NERVOUS SYSTEM VIRUS INFECTION}

In clinical practice, we can make our diagnosis of CNS virus infection based on clinical manifestations, CSF changes, and corresponding serum or CSF virus antibody detection results and neuroimaging observations. Using epidemic encephalitis, which is insect-borne viral encephalitis, for example, it can easily be found in summer and autumn, which are the high-incidence seasons. The main pathological changes are visible on the thalamus, basal ganglia, brain stem and cerebellum, with the corresponding changes visible on MRI scans. High fever, lethargy, coma and epilepsy are the most common symptoms. Herpes simplex viral encephalitis often involves bilateral temporal lobes and frontal lobes, and makes the patient prone to epilepsy and mental symptoms. Herpes simplex viral encephalitis can be diagnosed easily by typical imaging observations [Figure 1]. Rabies encephalitis often has a history of dog or cat bite; some patients may have the first attack 10 years after a bite. Until date, its pathogenesis has not been explained. Beside the clinical manifestations, rabies encephalitis has some notable symptoms, such as laryngeal muscle cramp, hydrophobia, and neck stiffness and opisthotonos. Head imaging generally shows no obvious changes, and some cases of rabies encephalitis progress quickly. Patients may go into coma and then die within a few days.

Many cases of viral meningoencephalitis cannot be confirmed effectively by testing because of the variation in viruses and detection of new viruses. Without typical clinical manifestations, such cases are easily misdiagnosed.

Acyclovir, rather than ganciclovir, should be the first choice of antiviral treatment for viral encephalitis. According to the virus treatment principle, treatment should last at least 3-4 weeks; a few cases may need treatment for 8 weeks or more. When elevated transaminases occur during treatment, we should distinguish between a
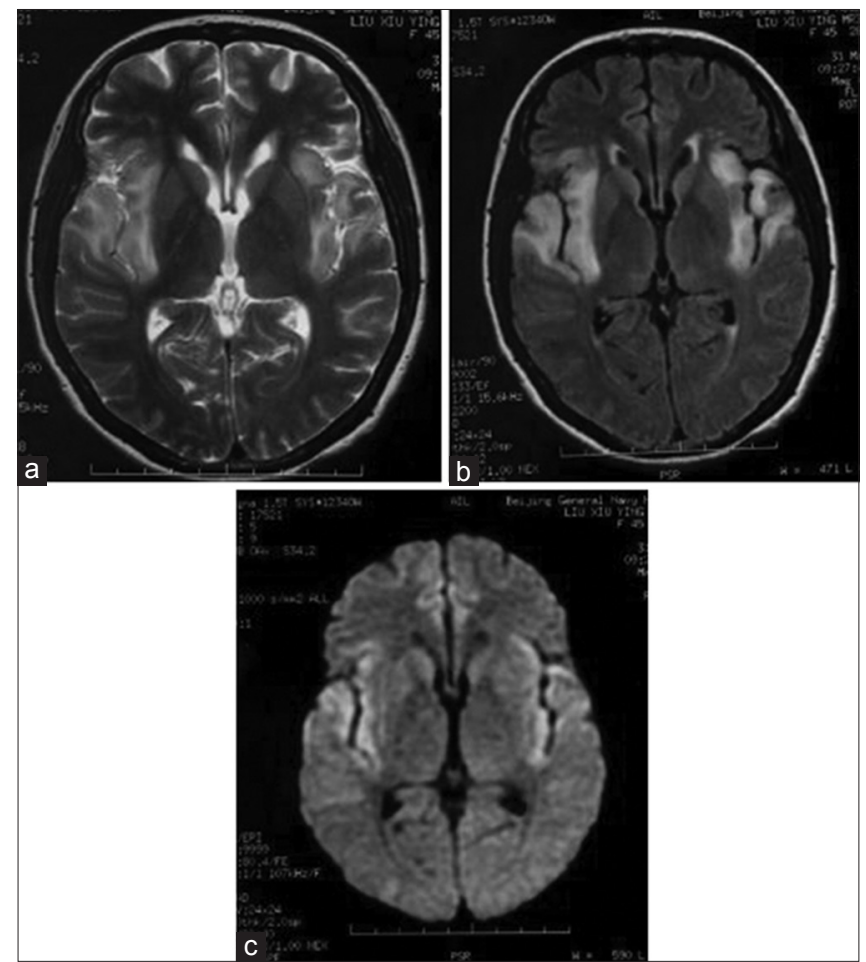

Figure 1: Magnetic resonance imaging representations of a herpes simplex virus encephalitis patient with intelligent obstacle and epilepsy seizure: high signal on T2-weighted image, fluid-attenuated inversion recovery (FLAIR) image and diffusion-weighted imaging (DWI) image on bilateral temporal lobe and insular lobe. (a) T2-weighted image; (b) FLAIR image; (c) DWI image 
temporary rise resulting from the virus infection, or the side-effects of the anti-virus medicine, and offer appropriate liver protective medication instead of immediately changing the medication.

\section{DIAGNOSIS OF CENTRAL NERVOUS SYSTEM PARASITIC AND FUNGAL INFECTIONS}

Parasitic infection has regional and seasonal features. For example, brain-type lung fluke (infected by eating raw or undercooked crab or crayfish) and Lyme disease (infected by tick bite) are more common in the Northern regions of China, while sparganosis (infected by playing with or eating frog) and amebic meningoencephalitis (infected by often swimming in a warm, muddy or dead freshwater ditch) are more common in the Southern regions of China. Hydatid disease is more common in the pastoral herders. At present, neurocysticercosis is relatively rare because constantly strengthened pork quarantine and dramatically improved local health conditions have greatly diminished pork infected by tapeworm. Different types of parasitic infections bear their own imaging characteristics. For instance, the head section and apparent body wall of a tapeworm could be distinguished clearly in images of cerebral cysticercosis infection, and the migration of "tunnel-like" lesions in the brain parenchyma are visible in images of sparganosis infection. All the above parasitic infections could be definitively diagnosed by specific antibody tests.

Fungal infection has gradually increased in recent years, mainly due to the increase in acquired immunodeficiency syndrome (AIDS) infection, transplant surgery and drug resistance to fungal medication. Certainly, Cryptococcus neoformans infection is still the most common while Aspergillus and Mucor infection of the nervous system is relatively uncommon. Fungal infection is generally an opportunistic infection and is not directly related to contact with pigeons (doctors often ask patients, whether they raise pigeons). Cryptococcal fungus also exists in the nasal passages of healthy subjects, but is usually not pathogenic. Cryptococcal infection usually occurs in subjects with a weakened or deficient immune system, such as in those with cancer or AIDS, or as a result of long-term use of immunosuppressive agents or hormones. Zhu et al. ${ }^{[5]}$ have reported that there was no decline in immune function in a patient infected with Aspergillus, in spite of the presence of brain-occupying lesions caused by Aspergillus infection. The clinical manifestations of this case resemble those of another 93 cases reported by Antinori et al., ${ }^{[6]}$ of which
$55.9 \%$ (52 cases) showed no decline in immune function and no predisposing factors. Therefore, fungal infections can also occur in people with normal immune function. It is not easy to distinguish deep brain-occupying lesions of granuloma formation from brain tumors and abscesses. Such cases require a diagnostic approach that combines CSF examination with bacteria and fungi examination, analysis of pathogens by incubation, and polymerase chain reaction testing. Brain radiation therapy or excision surgery should not be performed blindly before a clear diagnosis is made, otherwise the outcome will be misdiagnosis or, even worse, the spread of fungi. At present, the main treatment for fungal infections is by use of, for example, liposomal amphotericin B, fluconazole and voriconazole, which exhibit greater efficacy, safety and fewer side-effects than both amphotericin B and allicin.

\section{DIAGNOSIS OF PRION DISEASE}

Creutzfeldt-Jakob disease (CJD) is one type of prion disease - a molecular conformational disease caused by deposition of abnormal prion protein $\left(\mathrm{PrP}^{\mathrm{sc}}\right)$ - in which the structure of the normal prion protein PrPc changes, in neurons. Prion diseases, also known as "transmissible spongiform encephalopathies", are a class of CNS degenerative encephalopathies that can infect both animals and human beings with a long incubation period and a $100 \%$ mortality rate. In addition to CJD, human prion diseases include fatal familial insomnia, Kuru and Gisborne Terman-Strauss syndrome (GerstmannSträussler-Scheinker syndrome). The most common human prion disease is sporadic CJD, the incidence of which seems to have increased in recent years.

The typical symptom triad of CJD is progressive dementia, ataxia and myoclonus. Clinical manifestations can be divided into three stages. The early stage is characterized by weakness, fatigue, difficulty in concentrating, and memory loss. The interim stage (dementia-spasticity) is characterized by memory disorders, personality changes and dementia, and it can also be associated with aphasia and agnosia. Two-thirds of patients may exhibit myoclonus, and a series of symptoms may occur successively or alternately in this period owing to cortical, extrapyramidal, pyramidal or cerebellar (alternating or damaged) disease. At the late stage, urinary incontinence, akinetic mutism or decorticate rigidity arise. Diagnosis is confirmed by rapid progression of recent memory impairment, without symptoms of infection. Imaging, especially diffusion-weighted imaging and 
fluid-attenuated inversion recovery (FLAIR) pulse sequences, often reveals ribbon-like lesions along the cerebral cortex lesions, which is a very characteristic feature [Figure 2a and b]. However, at the late stage, brain atrophy and ventricular dilatation in patients is extremely severe, and ribbon-like lesions are no longer evident [Figure 2c and d]. White blood cells may also become visible in the CSF of the CJD patients. For instance, a virus or other infectious encephalitis cannot be confirmed until 10-30 white blood cells are found in the CSF. Until date, there has been no case in which the disease has been transmitted between patients, their family members and medical staff in China.

\section{DIAGNOSIS OF CENTRAL NERVOUS SYSTEM SYPHILIS INFECTION}

Recently, the incidence of syphilis - a disease caused by infection with Treponema pallidum - has escalated, with an increase in the incidence of syphilis infection of the CNS. Nervous system syphilis can be classified as, for example, asymptomatic neurosyphilis, syphilitic meningitis or myelomeningitis, syphilitic brain or spinal cord vasculitis, syphilis of the brain parenchyma (including polyparesis, tabes dorsalis and
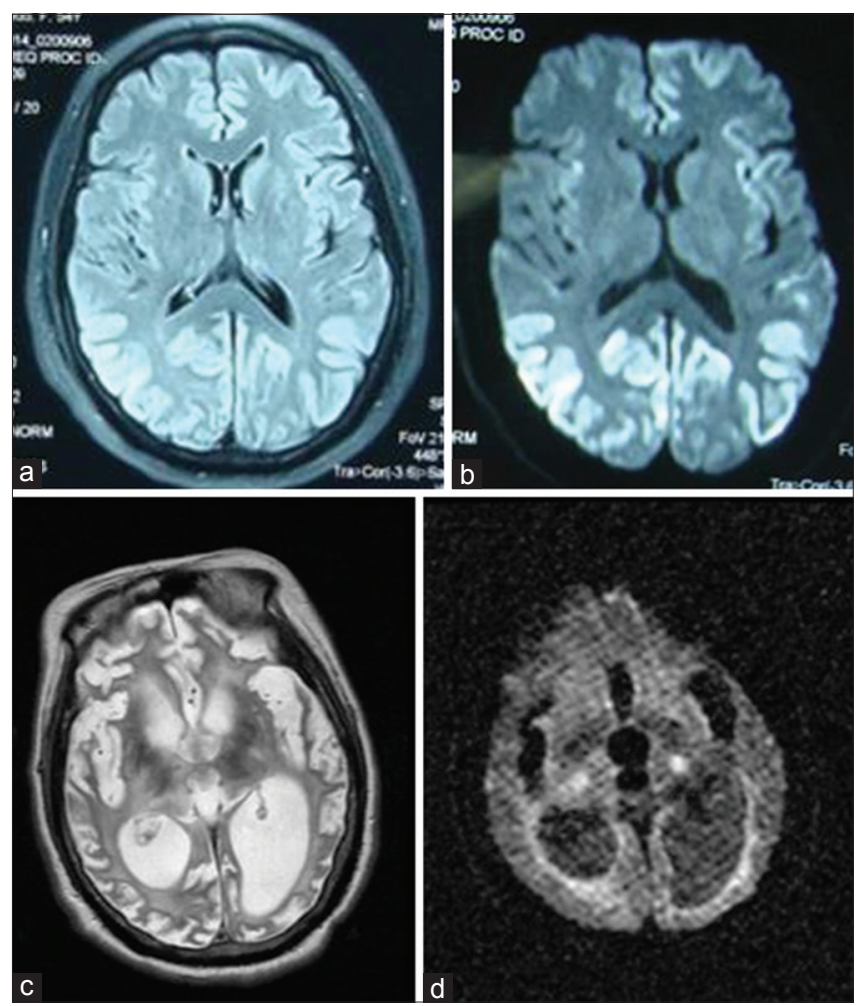

Figure 2: $(a$ and b) MRI representations of a sporadic Creutzfeldt-Jakob disease patient: high signal on both fluid-attenuated inversion recovery image and diffusion-weighted imaging (DWI) (2009-6-16). (c and d) T2-weighted image and DWI image of the same patient in a persistent vegetative state (2011-7-13): serious encephalatrophy with an obvious increase in ventricular volume syphilitic retrobulbar neuritis), syphilitic gumma, or acute inflammatory polyradiculoneuritis. Polyparesis usually occurs in patients aged 35-45 years with a long incubation of between several years and 20 years. With the insidious onset, the main symptom of polyparesis is progressive memory loss, which is easily misdiagnosed as Alzheimer's disease. At the early stage, polyparesis patients experience personality changes, anxiety, and emotional volatility, which can easily be misdiagnosed as depression.

In most cases, syphilitic antibody is the positive in serum, the white blood cells in the CSF are moderately elevated, and the protein level is also slightly increased, but a toluidine red unheated serum test and T. pallidum particle agglutination assay test can reveal normal results in a few cases. Imaging observations reveal brain atrophy, mainly in the hippocampus of the temporal lobe, and ventriculomegaly. ${ }^{[7]}$ There is a difference in antisyphilitic treatment between polyparesis and general syphilis. For polyparesis patients, treatment duration time is 6 months to 1 year, sometimes even longer, which is longer than that for general syphilis patients. Improvement in symptoms varies considerably between patients depending on when the disease is first diagnosed.

Besides the infections described above, there are other CNS infections with typical clinical characteristics, such as human immunodeficiency virus, Brinell bacillus infection, Whipple's disease, Guangzhou Angiostrongylus disease, and malaria. Such diseases can be diagnosed by the application of appropriate tests. It should be noted that, alongside the development in clinical practice, clinical viewpoints vary. For example, we used to think that parasitic infection in the brain would cause an increase in the eosinophil count in the CSF, but actually, in most parasitic infections of the brain, the eosinophil count does not increase (except for Guangzhou Angiostrongylus disease), and the eosinophil count in the peripheral blood was not elevated or even mildly elevated. A diagnosis of either eosinophilia or Churg-Strauss syndrome should be considered for patients with an elevated eosinophil count in their peripheral blood and fever. Diagnoses should be made with caution for patients with viral meningitis and no identified pathogen, but with normal electroencephalography, brain MRI scan and CSF, with reference to the patient's medical history and a careful consideration of the neurological examination, rather than reaching a conclusion based on only the results of a laboratory examination. Attention should also be paid to the differential diagnosis of immune-mediated 
encephalitis or autoantibody-mediated encephalitis (such as N-methyl-D-aspartate receptor encephalitis and anti-voltage-gated potassium channel antibody-associated encephalitis). ${ }^{[8]}$

\section{CONCLUSION}

More attention should be paid to the screening of CNS infection. We need to understand the geographical distribution, epidemic season and living history of all the pathogens, pathogenic pathways and pathogenic mechanisms and so forth. Careful consideration should also be given to the clinical history and the physical examination. Only in this way can we gain a better understanding of all the processes involved in CNS infection and provide a theoretical basis for appropriate treatments in the future.

\section{REFERENCES}

1. Mathers C, Boerma T, Ma Fat D. The Global Burden of Disease: 2004 Update. Geneva, Switzerland: World Health Organization; 2008. p. 1-146.
2. Bartzatt R. Tuberculosis infections of the central nervous system. Cent Nerv Syst Agents Med Chem 2011;11:321-7.

3. Patkar D, Narang J, Yanamandala R, Lawande M, Shah GV. Central nervous system tuberculosis: pathophysiology and imaging findings. Neuroimaging Clin N Am 2012;22:677-705.

4. Zhao G, Du F. Diagnostic strategy of tuberculous meningitis. Chin $J$ Contemp Neurol Neurosurg 2013;13:1-4. (in Chinese)

5. Zhu WJ, Qiu H, Tan JP, Dong Z, Huang XS, Liu CL, Zhao XS. Invasive aspergillosis involving cavernous sinus and orbital apex in an immunocompetent youth: a case report and literature review. Chin J Neuroimmunol Neurol 2014;20:85-8. (in Chinese)

6. Antinori S, Corbellino M, Meroni L, Resta F, Sollima S, Tonolini M, Tortorano AM, Milazzo L, Bello L, Furfaro E, Galli M, Viscoli C. Aspergillus meningitis: a rare clinical manifestation of central nervous system aspergillosis. Case report and review of 92 cases. $J$ Infect 2013;66:218-38.

7. Qiu F, Qi XK, Xiong B, Liu JG, Wang W. The clinical and imaging features of general paresis of insane. J Brain Nerv Dis 2006;14:213-4. (in Chinese)

8. Laura W, Paul E, Terry K. Encephalitis. Pediatr Emerg Care 2013;29:235-44.

Cite this article as: Qi XK. Diagnosis and therapy of rare central nervous system infections. Neuroimmunol Neuroinflammation 2014;1(1):8-12.

Source of Support: Nil. Conflict of Interest: No.

Received: 31-03-2014; Accepted: 29-05-2014 